\title{
Influence of Geometry and Velocity of Rotating Solids on Hydrodynamics of a Confined Volume
}

\author{
Ignacio Carvajal-Mariscal, ${ }^{1}$ Cesar A. Real-Ramírez, ${ }^{2}$ Florencio Sánchez-Silva, ${ }^{1}$ \\ Francisco Cervantes de la Torre, ${ }^{2}$ and Jesús González-Trejo ${ }^{2}$ \\ ${ }^{1}$ Instituto Politécnico Nacional, ESIME, UPALM, 07738 Mexico City, Mexico \\ ${ }^{2}$ Universidad Autónoma Metropolitana, San Pablo 180, Reynosa Tamaulipas, 2200 Mexico City, Mexico
}

Correspondence should be addressed to Cesar A. Real-Ramírez; carr@correo.azc.uam.mx

Received 19 May 2017; Revised 12 September 2017; Accepted 11 October 2017; Published 9 November 2017

Academic Editor: Anna Vila

Copyright ( 2017 Ignacio Carvajal-Mariscal et al. This is an open access article distributed under the Creative Commons Attribution License, which permits unrestricted use, distribution, and reproduction in any medium, provided the original work is properly cited.

\begin{abstract}
Three cylinder-based geometries were evaluated at five different rotating speeds $\left(\omega=20.94,62.83,94.25,125.66\right.$, and $\left.157.08 \mathrm{rad} \cdot \mathrm{s}^{-1}\right)$ to obtain the fluid flow pattern in nonsteady conditions. Two of the models were modified at the lower region, also known as tip section, by means of inverted and right truncated cone geometries, respectively. The experimental technique used a visualization cell and a Particle Imaging Velocimetry installation to obtain the vector field at the central plane of the volume. The Line Integral Convolution Method was used to obtain the fluid motion at the plane. In addition, the scalar kinetic energy and the time series were calculated to perform the normal probability plot. This procedure was used to determine the nonlinear fluid flow pattern. It was also used to identify two different flow regimens in physical and numerical results. As the rotation speed increased, the turbulent regions were placed together and moved. The process makes experimental observation difficult. The biphasic and turbulence constitutive equations were solved with the Computational Fluid Dynamics technique. Numerical results were compared with physical experiments for validation. The model with the inverted truncated cone tip presented better stability in the fluid flow pattern along the rotation speed range.
\end{abstract}

\section{Introduction}

A system with a rotating solid in a confined volume is common in several industrial processes, like mixing, electrochemistry, and others [1-7]. The hydrodynamics in the confined volume of this kind of systems present a highly turbulent flow. To characterize the flow pattern, among other variables, three relevant parameters on the process have been identified: the distance ratio between the solid and the walls of the confined volume, the geometry of the cylinder, and the rotating speed.

The interaction of the fluid with the rotating solid is an essential factor that determines the whole process. Thus, the geometry of the solid has been changed to fulfill the specific requirement of a process $[8,9]$. But, most of these modifications were based on the results obtained by the process itself and not by the investigation of the phenomenon. Before taking the decision to use any given geometry, there should be a careful and thorough study of the hydrodynamics in the confined volume [10].

At the same time, the rotating speed of the solid has an important role in the process. In some cases, a low rotation speed of the solid is needed, while in others a higher rotation provides the necessary conditions to achieve the given purpose $[5,11,12]$. It was found that high velocities of the rotating solid induce the formation of an air bubble around the solid. Consequently, the hydrodynamics of the whole confined volume change. Therefore, research on the influence of the geometry and velocity of the rotating solid has to be performed.

To understand the hydrodynamics of this kind of systems, an experimental investigation should be carried out. It should imply the use of a nonintrusive technique, such as the Particle Image Velocimetry (PIV). This technique has been 


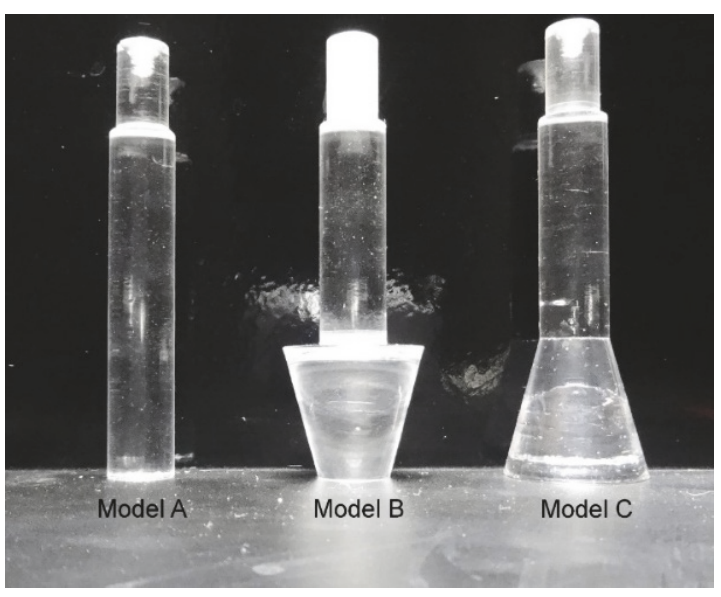

(a)

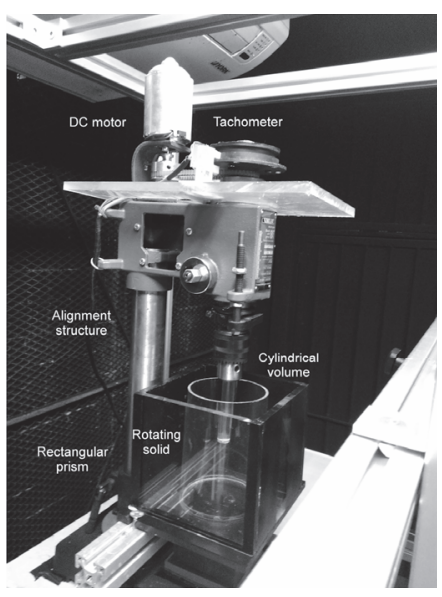

(b)

Figure 1: Physical experiment setup. (a) Models of translucent solid bodies. (b) Experimental rig with rectangular prism cell and rotation body.

successfully used to investigate complex phenomena [13-19] and thanks to its accuracy, it is a reference to validate the numerical results. Finally, to complement the results of the experimental research, a numerical study would be necessary.

Because of the complex hydrodynamics in the confined volume, the numerical study had to be performed during a nonsteady state to obtain the transient behavior of the phenomenon and the three-dimensional properties of the flow, as it has been noticed in previous works [10]. A two-dimensional approach could lead to the obtaining of errors in the flow pattern determination due to the simplification of the system.

One of the main applications of this work is to study the hydrodynamics inside electrochemical cells with a rotating electrode. The bell-shaped cylinder was studied since the 1960s and electrodes with this shape are still used. Surprisingly, studies of electrodes with a shape of an inverted cone have never been reported. Therefore, it is important to conduct the analysis of the hydrodynamics behavior of a system by using a cylinder with this kind of shape since the geometry of the electrochemical cells has changed in recent years. Some designs reduced the dimensions of the cells to work with lower rotation speeds. Other designs have isolated the working electrode to minimize the effect of counter electrode and the reference electrode [20].

In this work, experimental research using the PIV technique and the numerical simulations using the CFD technique were developed to obtain the hydrodynamics information in a cylindrical confined volume for three distinct shapes of the rotating solid and angular velocities from 20.94 to $157.08 \mathrm{rad} \cdot \mathrm{s}^{-1}$.

\section{System Description}

Figure 1(a) shows the three models of cylinders used in this study. All of them have a cylindrical upper part with 0.011 meters of external diameter and 0.02 meters of length; the "offside" is used to connect the rotating mounting.
Model A is a solid cylinder with a 0.015 -meter external diameter, $d$, and $5 d$ of length. It is called "midst."

Model B has a midst with an external diameter of $1 d$ and $3 d$ of length. The lower part, also called "tip," has an inverted truncated cone shape with $1 d$ of base and $2 d$ of top surface with an altitude of $2 d$, as shown in Figure 1(a).

Model C has a similar midst as model B. The tip has a right truncated cone shape with $2 d$ of base and $1 d$ of top surface with an altitude of $2 d$, as shown in Figure 1(a).

The confined volume has a cylindrical shape and was constructed in translucent acrylic of 0.002 meters of thickness. The internal diameter of the volume is $6 d$ and the altitude is $10 d$. Each cylinder was located at the center of the cylindrical volume at a distance of $5 d$ to its base.

All three cylinders were coupled to a rotating mounting that is aligned with a metallic drill. The rotating mounting is driven by a DC motor controlled by a potentiometer. In order to get a constant rotating speed during the test, the potentiometer was connected to an optical tachometer mounted in the upper zone of the experimental rig, as shown in Figure 1(b).

\section{Mathematical Model}

The constitutive equations of the cylindrical volume and a centered cylinder at constant rotating speed were solved based on the CFD technique with the Volume Finite (VF) method. The $\kappa-\varepsilon$ turbulence model was used to calculate the vectorial field inside the three-dimensional numerical model based on the physical experiment.

The turbulent kinetic energy $\kappa$ and its rate of dissipation $\varepsilon$ were obtained from the transport equations. To model the interface between the water and air phases, the Volume of Fluid (VOF) model was used in the case of two or more immiscible fluids. This model is appropriate due to the phases or fluids being at rest. The inertial forces caused by the swirl of the centered cylinder govern the hydrodynamic 
phenomenon. The working fluids are water as primary phase and air as secondary phase.

The model is based on a surface tracking technique and is applied to a nonstructured and fixed Eulerian mesh. The tracking of the interfaces between the phases is obtained by using the continuity equation for the volume fraction of both phases.

The continuity equation for multiphase flow is

$$
\begin{aligned}
& \frac{1}{\rho_{q}}\left[\frac{\partial}{\partial t}\left(\alpha_{q} \rho_{q}\right)+\nabla \cdot\left(\alpha_{q} \rho_{q} \vec{v}_{q}\right)=S_{\alpha_{q}}\right. \\
& \left.+\sum_{p=1}^{n}\left(\dot{m}_{p q}-\dot{m}_{q p}\right)\right],
\end{aligned}
$$

where $\dot{m}_{q p}$ is the mass transfer from phase $q$ to phase $p$ and $\dot{m}_{p q}$ is the mass transfer from phase $p$ to phase $q$. The term $S_{\alpha_{q}}$ is defined as zero. The term $\alpha_{q}$ is the phase value of the $q$ th volume fraction. The term $\rho_{q}$ is the density value of the $q$ th volume fraction. The values of 998.2 and $1.204 \mathrm{~kg} \cdot \mathrm{m}^{-3}$ have been used for the water and air phases. The term $\nabla$ is the divergence operator. The volume fraction equation was not solved for the primary phase; instead, it was computed by the following condition [21]:

$$
\sum_{q=1}^{n} \alpha_{q}=1
$$

The term $\alpha_{q}$ is the phase value of the $q$ th volume fraction to phase $n$. The volume fraction equation (see (1)) was solved by using the explicit time formulation. Initial conditions were defined based on measurements from the physical experiment. The velocity of the water at $t=0$ is zero. The rotation velocity of the cylinder was constant in each of the simulations. The number of cylinders was three and the number of rotation velocities was five, $\omega=20.94,62.83,94.25$, 125.66 , and $157.08 \mathrm{rad} \cdot \mathrm{s}^{-1}$. The number of simulations was 15 . The volume fraction is discretized in the following form:

$$
\begin{gathered}
\frac{\alpha_{q}^{n+1} \rho_{q}^{n+1}-\alpha_{q}^{n} \rho_{q}^{n}}{\Delta t} V+\sum_{f}\left(\rho_{q}^{n+1} U_{f}^{n+1} \alpha_{q, f}^{n+1}\right) \\
=\left[S_{\alpha_{q}}+\sum_{p=1}^{n}\left(\dot{m}_{p q}-\dot{m}_{q p}\right)\right] V .
\end{gathered}
$$

$n+1$ is the index for a new time step, $n$ is the index for the present time step, $\alpha_{q, f}$ is the phase value of the $q$ th volume fraction, and the term $\rho_{q}^{n}$ is the density value of the $q$ th volume fraction in the present time step. The term $\dot{m}_{q p}$ is the mass transfer from phase $q$ to phase $p$ and $\dot{m}_{p q}$ is the mass transfer from phase $p$ to phase $q$.

The term $S_{\alpha_{q}}$ is the user-defined source term, and it is defined as zero. $V$ is the volume of the cylinder and $U_{f}$ is the volumetric flux through the phase, based on normal velocity. Since the volume fraction at the current time step is calculated based on known quantities of the previous time step, the formulation does not require an iterative solution of transport equation during each time step [21].

Numerical models are solved with a single set of momentum equations, and the two phases of the system are tracked as the volume fraction of each of the fluids throughout the domain [21].

$$
\begin{aligned}
& \frac{\partial}{\partial t}(\rho \vec{v})+\nabla \cdot(\rho \vec{v} \vec{v}) \\
& \quad=-\nabla p+\nabla \cdot\left[\mu\left(\nabla \vec{v}+\nabla^{T}\right)\right]+\rho \vec{g}+\vec{F}
\end{aligned}
$$

The fields for all variables and properties are shared and represent volume-averaged values. The term $\rho$ is the density value, the term $\vec{v}$ is the velocity vector, the term $\nabla$ is the divergence operator, the term $p$ is the pressure with a 1.013 . $10^{5} \mathrm{~Pa}$ value, the term $\mu$ is the dynamic viscosity, $1.983 \cdot 10^{-5}$ and $10^{-3} \mathrm{~Pa} \cdot \mathrm{s}$ were the values of the air and water phases, the term $\vec{g}$ is the gravity vector with a $9.8 \mathrm{~m} \cdot \mathrm{s}^{-2}$ value, and $\vec{F}$ is the force vector.

In addition, the nonslip condition was imposed in all the internal walls. This condition was also considered in the cylinder walls. Besides, the pressure inlet condition was imposed in the upper wall of the system. All the numerical simulation initiates at rest.

\section{Operation Conditions}

The PIV technique was used since it makes it possible to investigate the flow behavior without any disturbance due to the measurement process.

The PIV system consisted of a CCD camera with a resolution of 1,632 $\times 1,200$ pixels, a double-pulsed Nd:YAG Laser source with a wavelength of $532 \mathrm{~nm}$ and $150 \mathrm{~W}$ maximum output, a synchronizer, and a work station. Measurements were performed and data processed by using a Dantec Dynamics TR PIV System and Dynamic Studio Software. The laser pulses and camera were triggered by means of a synchronizer; the trigger rate was set at 200 pulses per second.

To observe the fluid motion, the water was seeded with neutrally buoyant silver-coated glass spherical particles, $10 \mu \mathrm{m}$ diameter. The measurement plane was illuminated by using $40 \%$ of the maximum laser energy output. The time interval between pulses was $0.00119 \mathrm{~s}$ for all measurements. The thickness of the laser sheet illuminating the measurement plane was of 0.0015 meters approximately. The movement of the particles was recorded using a CCD camera. A frame-to-frame adaptive technique was used to calculate raw displacement vectors.

In order to reduce the optical image noise, the cylindrical volume was put inside a cube opened at the top; it is called "visualization cell," as shown in Figure 1(b). The visualization cell is made of 0.009-meter thick acrylic and has a length of 0.014 meters. The base and three of the four walls have been painted black, but one of the walls has an unpainted 0.03-meter wide vertical fringe to allow the laser sheet to pass through the cylindrical volume illuminating its central plane. The remaining wall has not been painted, to capture the images with the high-speed camera. 
The visualization cell and the cylindrical volume were filled with water to a height of $7 d$. The system was at rest at the beginning of the test; the potentiometer was turned on and operated for 300 seconds to stabilize the flow pattern conditions. Once the rotation speed is maintained, the PIV system initializes to obtain 300 seconds of the process.

Data reported herein consist of $203 * 149$ vectors representing the plane region within the cylindrical volume. Postprocessing of the data was performed on the 1000 pairs of images on each rotating speed, to determine mean velocities and relative uncertainties to $95 \%$ confidence and RSS levels.

The cylinder has five different rotation speeds that represent different flow behavior; they are almost equally spaced. $\omega=20.94,62.83,94.25,125.66$, and $157.08 \mathrm{rad} \cdot \mathrm{s}^{-1}$. The initial rotation speed is considered to obtain turbulence and a quasi-stable state inside the volume. With the final rotation speed, significant turbulence was obtained. However, the free surface has no appreciable influence in the fluid flow pattern.

The numerical model has the same geometrical dimensions as the cylindrical volume showed in Figure 1(b). The model considers the air and the water phases, both at rest and at $t=0$ seconds. The discretization process consists in a global control on the minimum size of $1.1 \cdot 10^{-5}$ meters on each element. Also, the scalar size function is defined by a growth rate of 1.2 to obtain a regular mesh in the control volume and to avoid the divergence due to the difference between the sizes of the elements.

The discretization mainly consists in triangular elements with ten rows of boundary layers close to the walls of the cylindrical volume and the solid cylinder. The scalar aspect ratio was defined as 10 and a growth rate of 1.2 at the lateral walls. The total number was approximately $1.7 \cdot 10^{6}$ elements in every model.

A zero wall shear stress at the walls was defined. Also, a nonsteady condition was calculated for the numerical model. The initial time step with a scalar value of $5 \cdot 10^{-4}$ and 150 iterations per time step were defined. The scaled residual of different variables was considered as convergence criterion and all the residual values were less than $10^{-5}$.

The convergence was obtained after a maximum number of 150 iterations in each time step and a growth rate of 2 was defined to change the size of the time step. The calculation of the Courant number was less than 2, to continue with the calculations. The total numbers of calculations allow obtaining a 300-second process.

\section{Results}

With the PIV technique, images of moving particles in a confined volume via an illuminated plane by a laser source are obtained [22]. In this work, with the image processing software, the velocity field was calculated by means of the adaptive correlation method [23]. A vector-masking filter was applied to remove the information outside the control volume. The Line Integral Convolution Method was applied to describe the trajectory of the particles more clearly [24].

Figures 2(a), 2(b), and 2(c) show the flow behavior at $\omega=20.94 \mathrm{rad} \cdot \mathrm{s}^{-1}$ for the three models of cylinders. Because of the low rotation speed, a similar behavior was observed in the three cases. The upward flow around the lateral walls of the cylinder interacted with the free surface of the working fluid. The flow generated recirculation zones form a toroidal structure, and then the flow was redirected downwards. Two recirculation zones were generated close to the lateral walls. The region below the cylinder presented a particular flow behavior; the flow moved upwards, hit the base of the cylinder, and moved rather to the right.

In model $\mathrm{A}$, it was observed that there was no considerable turbulence below the base of the cylinder, but the upward flow passed around its lateral walls. Near the base of the confined volume, the flow moved horizontally. However, due to the swirl of the cylinder, the flow changed its direction to the vertical component up to the base. A small part of the flow moved to the left lateral wall of the cylinder.

Figure 2(b) shows an inverted cone-shaped tip at $\omega=$ $20.94 \mathrm{rad} \cdot \mathrm{s}^{-1}$; the upward flow around the inverted cone is close to the base. However, in the middle of the bases there is a horizontal flow that promotes the generation of greater vortex. The turbulent zone is close to the cylinder; yet the turbulence generated around the tip has grown and ascended to the free surface.

In model $\mathrm{C}$, the region below the base of the cylinder is preferably horizontal; however, as the flow went up, the streamlines moved vertically and returned to the horizontal component. The turbulent structures were located close to the lateral walls and moved downwards to form a recirculation zone near the lower left of the confined volume.

Figures 2(d), 2(e), and 2(f) show the Line Integral Convolution Technique applied for different models at $\omega=$ $62.83 \mathrm{rad} \cdot \mathrm{s}^{-1}$. In model $\mathrm{A}$, the region of interest is wider than $\omega=20.94 \mathrm{rad} \cdot \mathrm{s}^{-1}$ at the base of the cylindrical volume. It is possible to observe an upward flow direct to the right lateral wall of the solid cylinder and a horizontal flow to the base of the cell. This phenomenon is similar to that of model C, although it is wider.

We can see the notorious presence of an equivalent diameter vortex located in the lower left corner in Figure 2(f). In model $\mathrm{B}$, it is feasible to observe an upward flow at the base of the cone that directs to the right lateral wall of the cylinder.

At a rotation speed of $=94.25 \mathrm{rad} \cdot \mathrm{s}^{-1}$, model A presents an upward flow to the base of the cylinder. However, a small part of the flow moved to the lateral left wall of the solid cylinder and developed a vortex at the lower left corner of the confined volume. The rest of the flow moved to the lateral left wall of the volume.

In Figure 2(i), the region of upward flow and the vortices remained quasi-stable and well-developed. Greater vortices were observed. An upward flow was also noted from the left corner of the volume. Almost all the vortices close to the lateral walls have lower dimensions than in model $\mathrm{A}$ and model B. The flow behavior below the inverted truncated cone shape has a similar form as the solid.

Figures 2(j), 2(k), and 2(l) show the fluid flow behavior at $\omega=125.66 \mathrm{rad} \cdot \mathrm{s}^{-1}$, for the three cases. In model $\mathrm{A}$, the flow moves from the lower left corner to the base of the cylinder. Once the flow was near the base, it moved forward to the right 


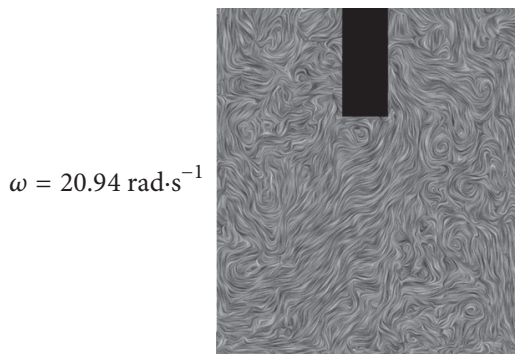

(a)

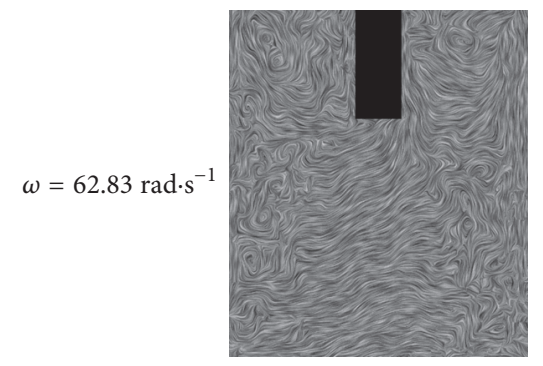

(d)

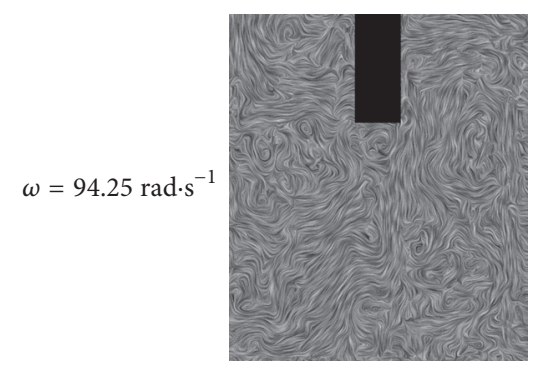

(g)

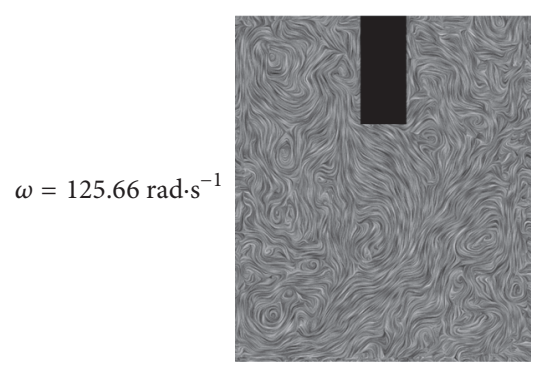

(j)

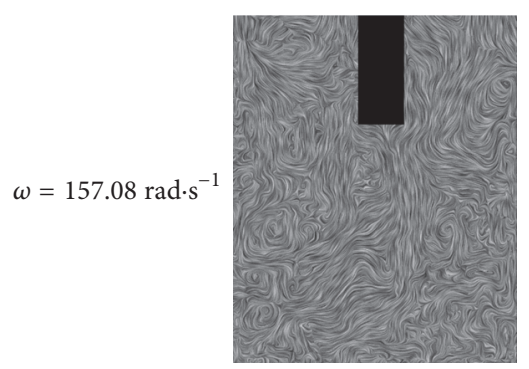

(m)

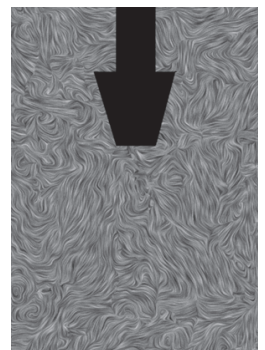

(b)

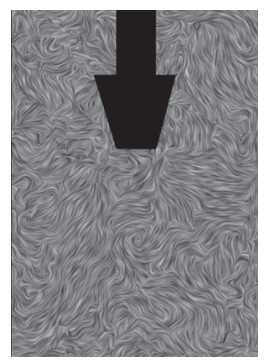

(e)

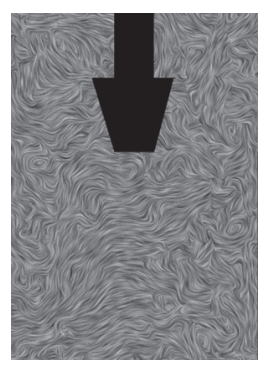

(h)

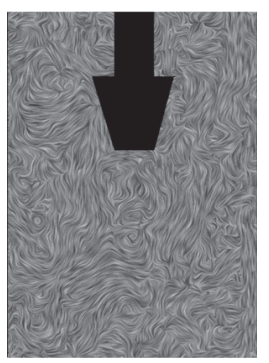

$(\mathrm{k})$

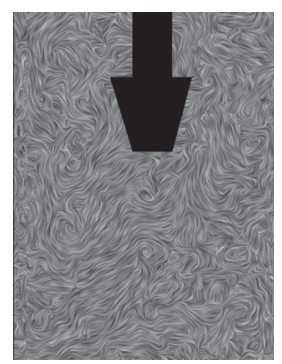

(n)

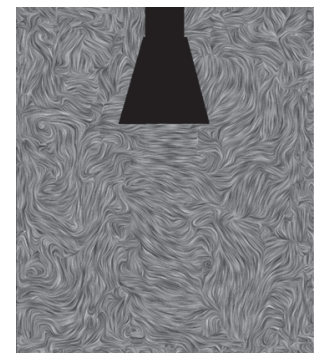

(c)

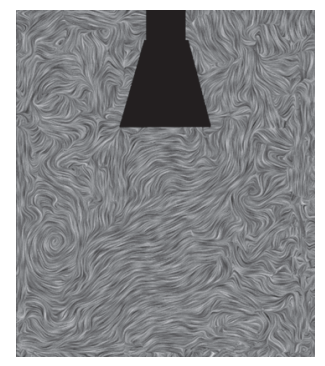

(f)

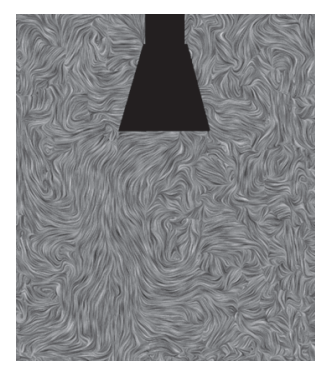

(i)

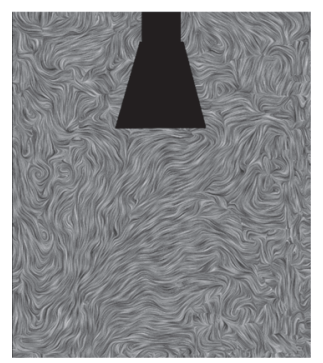

(l)

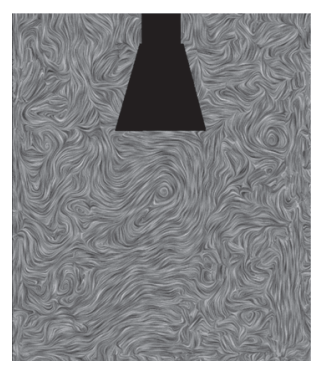

(o)

FIGURE 2: Line Integral Convolution Method for different models at different rotating speeds. 
lateral wall of the solid and continued to rotate in the toroidal structure around the cylinder. In this model, the vortices are not near the lateral walls. The turbulent flow moved to the center and directs the upward flow to the center. A vortex below the base of the cylinder that moved part of the flow to the left was also observed. Around the cylinder, the toroidal structure surrounds the cylinder. Part of the flow moved downwards and generated another turbulent zone below the base of the cylinder. The flow directed to the base and moved laterally to the center of the volume.

In model B, a turbulent flow behavior below the inverted truncated cone is observed. However, there is an upward flow at the center of the volume. There are turbulent structures that mixed each other. The toroidal structure around the cylinder occupies the volume near the cylinder and interacts with the flow below the base of the cylinder. However, it is possible to observe an upward flow below the base of the cylinder.

Several turbulent structures appeared on the plane, mixed with greater ones, and moved faster than previous rotation speed. The flow close to the solid was turbulent and complex to describe.

The flow behavior in model $\mathrm{C}$ is quasi-stable, and the width is similar to the truncated cone shape of the solid diameter. The flow was predominantly horizontal and had the same rotation as the base of the solid cone. The flow near the lateral walls moved away and joined a turbulent zone around the rotating cylinder.

The toroidal structure around the solid is not welldefined; however, part of the flow moved downward and reintegrated to other regions of turbulent flow. The vortices appeared to be vertically aligned below the base of the cylinder at this plane; however, the core of the vortex moved continuously.

Figures 2(m), 2(n), and 2(o) show the Line Integral Convolution Method applied to the three different models at $\omega=157.08 \mathrm{rad} \cdot \mathrm{s}^{-1}$. In model $\mathrm{A}$, the flow behavior has similar characteristics to the previous rotation speed at $\omega=$ $125.66 \mathrm{rad} \cdot \mathrm{s}^{-1}$; it also shows a vortex near the base of the cylinder. The width of the vortex has one equivalent diameter of the base of the cylinder. With this rotation speed, an upward flow, which ascends below the base of the solid and is forwarded to the lateral walls, is observed. The structures around the solid moved fast and the visualization of cores in the plane is difficult.

In model $\mathrm{B}$, at $\omega=157.08 \mathrm{rad} \cdot \mathrm{s}^{-1}$, the flow structure below the inverted, truncated cone had a similar flow behavior with a preponderant horizontal component. However, at the center of the base there was an upward flow.

The vortices around the solid move fast, they move downwards close to the lateral wall, and then they move horizontally, from left to right preferably. There are numerous vortices on the right lateral wall of the volume; their size is approximately a radius of the base of the cylinder.

In model $\mathrm{C}$, the flow direction in the base of the truncated cone changed from horizontal to vertical. In addition, it switched between right and left; the flow was divided in both directions. It is possible to observe that the flow fluctuated quickly. However, the flow behavior has a similar pattern to the truncated cone shape and the cores of the vortices have a similar radius to half of the base of the solid.

In Figure 2(n), we observe an upward flow from the base of the volume to the base of the solid. However, the flow changed rapidly to a horizontal flow direction.

According to the observations made from the physical experiment, when the rotation speed is low, the kinetic energy behavior may approach a given normal distribution. This means that the flow interacts with the walls of the cylindrical volume uniformly, the transient behavior is minimal, and the sizes and location of the vortices have no considerable changes.

The probability plot is a graphical technique used to know if a data set is close to a normal distribution [25]. If experimental data is plotted in a graph against a theoretical normal distribution, when the kinetic energy is distributed normally, it should be close to the straight line.

Although this technique is commonly used to obtain an associated correlation coefficient, the present study considers that the shape of the curve helps to describe a nonlinear and high turbulent behavior of the flow.

From the velocity field, it is possible to integrate the velocity magnitude of each illuminated particle to obtain the scalar value of the kinetic energy in the central plane of the confined volume. The kinetic energy was calculated to quantify the motion of the particles within the plane. This calculation allows knowing the turbulence in the system.

Once the kinetic energy data was obtained from the image processing technique, it is possible to get the probability distribution plot. The $x$-axis of the plot normalized a normal distribution behavior; meanwhile, the $y$-axis of the plot normalized the experimental data and obtained the dimensionless and normalized axes in Figures 3(a), 3(b), and 3(c).

The data were plotted against an "ideal" distribution in such a way that the points should form (approximately) a straight line. In the ideal case, the fluid flow pattern inside the cylindrical volume was symmetrical and quasi-stable. Even though the confined volume and the models of cylinders are symmetrical to a longitudinal central axis, the flow behavior inside the volume is not symmetric. This can be explained because the rotation of the cylinder promotes an interaction that "accumulates" in the system and modifies time.

Visually, the probability plot shows a nonlinear pattern in every rotation speed in model A. However, when the rotation speed is $\omega=20.94 \mathrm{rad} \cdot \mathrm{s}^{-1}$, the flow has a behavior closer to that of a normal distribution. It can also be mentioned that the behavior is similar at almost all rotation speeds except at $\omega=62.83 \mathrm{rad} \cdot \mathrm{s}^{-1}$ when the yellow colored line moves away from the normal distribution line and forms an S-like pattern shaped curve.

If we draw a vertical line at $x=0.5$, it is possible to observe that there is no symmetry at any rotation speed and that the data points are concentrated on the left half of the plot. Moreover, the lower tail at any rotation speed shows a considerable increasing departure below the fitted line. Nevertheless, there is almost no existence of an upper tail; the experimental data accumulated above the fitted line. 

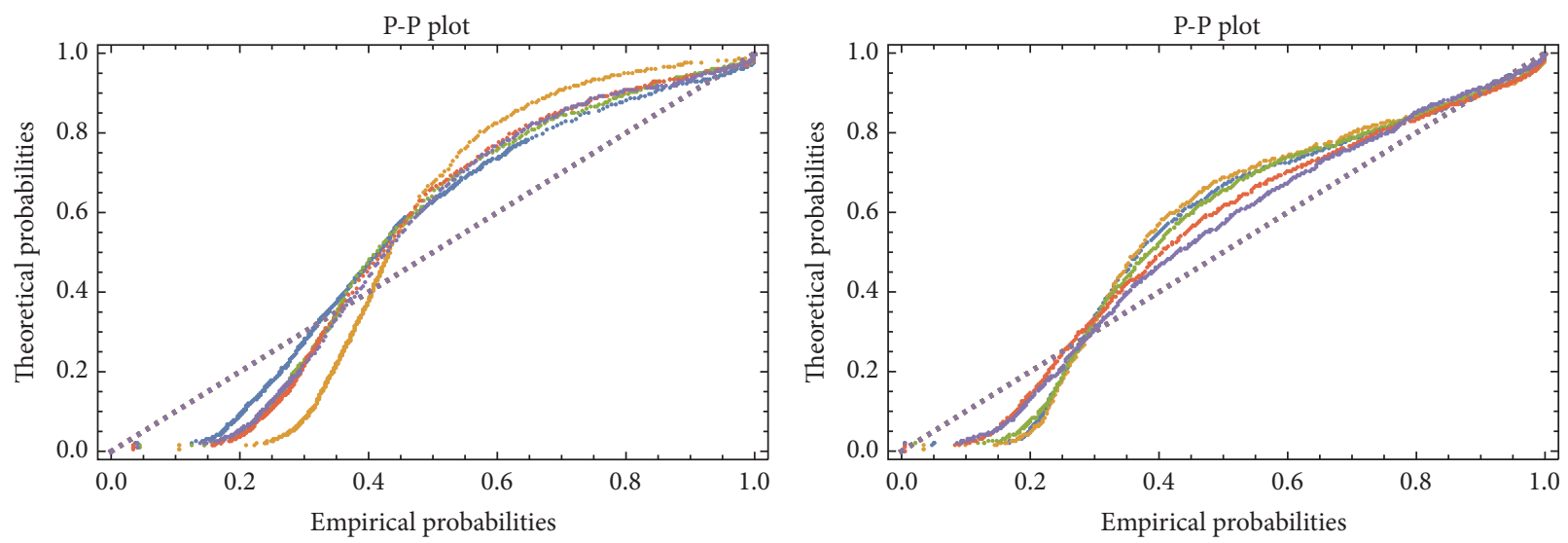

Model A

$$
\begin{aligned}
& \because \cdots \omega=20.94 \mathrm{rads}^{-1} \quad \cdots \omega \omega=125.66 \mathrm{rad} \mathrm{s}^{-1} \\
& \cdots \omega \omega=62.83 \mathrm{rads}^{-1} \quad \ldots \cdots \omega=157.08 \mathrm{rad} \mathrm{s}^{-1}
\end{aligned}
$$

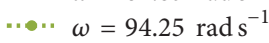

(a)

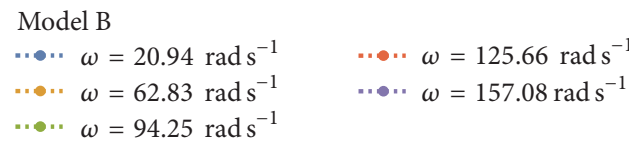

(b)

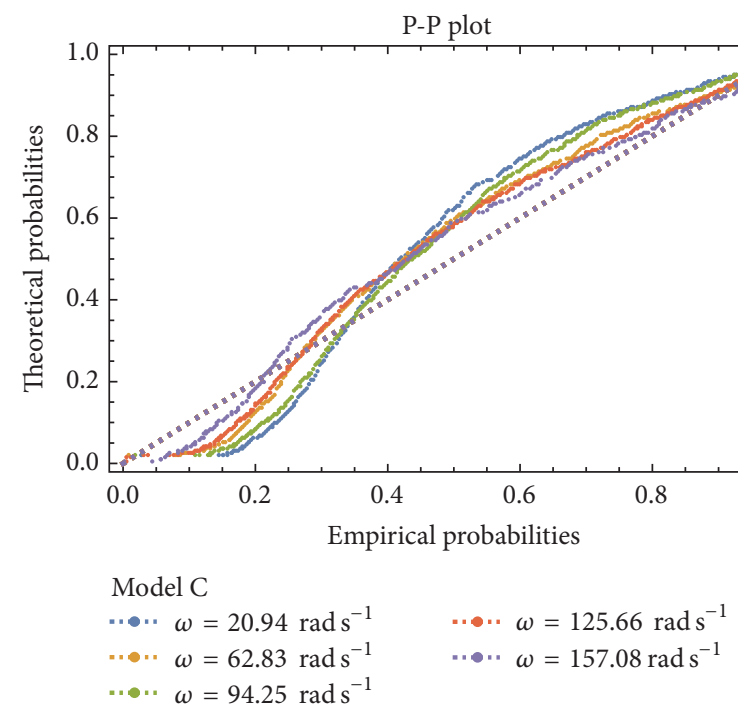

(c)

FIGURE 3: Different rotation speeds probability plot for three different models compared to an estimated normal distribution.

For the entire rotation speed range, model $\mathrm{B}$ shows a nonlinear and asymmetrical behavior. The curves were closer to the estimated normal distribution behavior than in model A. The lower tails are below the fitted line similar to model A; if we use a vertical axis in 0.3 , model $B$ in all rotating speeds is closer to a normal distribution than model $\mathrm{A}$.

In addition, for model $\mathrm{B}$ at $\omega=62.83 \mathrm{rad} \cdot \mathrm{s}^{-1}$ the yellow curve shows the greatest distance from the normal distribution line, while at $\omega=157.08 \mathrm{rad} \cdot \mathrm{s}^{-1}$ the purple line is closer to the normal distribution. For this angular velocity, the flow behavior is relevant. It would be expected that as the rotation speed increases the curve gets away from the normal distribution line. The behavior along the rotation speed range shows a progressive form. This finding could serve to obtain reproducibility at different flow regimes.
At $x=0.3$, the change between the experimental data below and above the normal distribution is shown. The pattern in which a majority of points are above the reference line means that the data set is left-skewed.

Model C has an S-shaped pattern that can be considered as a left-skewed data set. The point in which the curves intersect is also above the distribution line. The lower tail gets away from the line and the upper tail gets closer to the distribution. Even when the purple line at $\omega=157.08 \mathrm{rad} \cdot \mathrm{s}^{-1}$ is closer to the uniform distribution line and the blue line at $\omega=157.08 \mathrm{rad} \cdot \mathrm{s}^{-1}$ is the farthest curve, symmetrical behavior at different rotation speeds is not observed.

Figure 4 shows the numerical simulations where the Computational Fluid Dynamics (CFD) technique was used. The constitutive equations were solved by the Finite Volume 


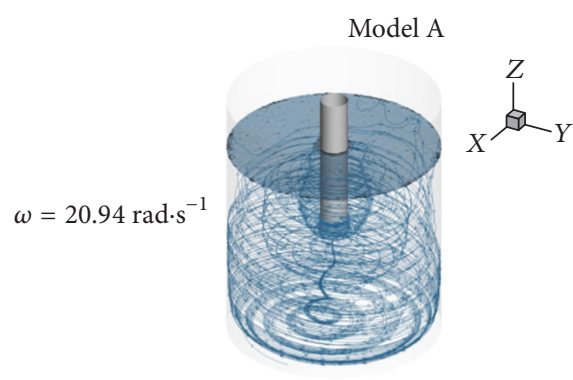

(a)

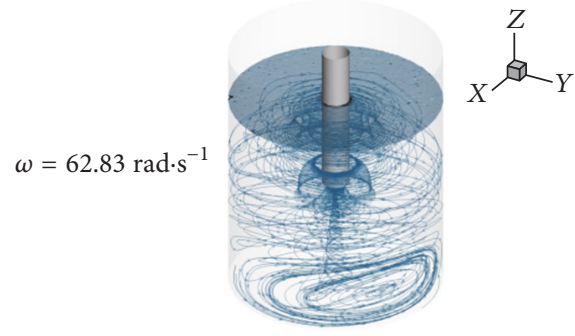

(d)

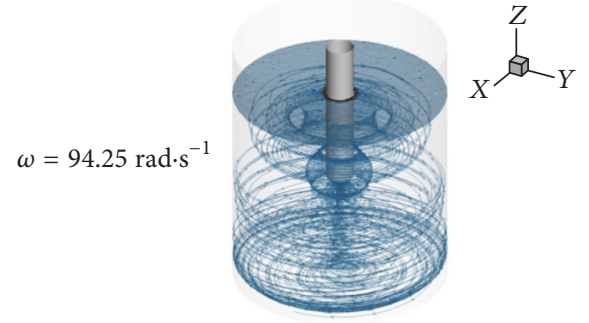

(g)

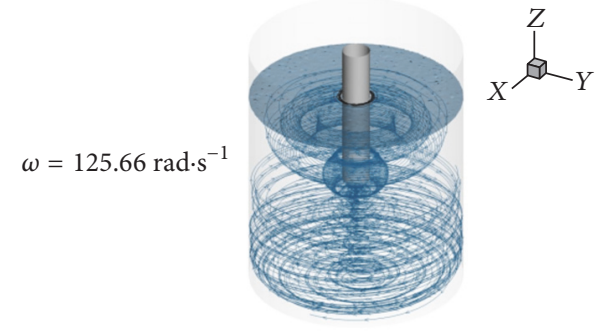

(j)

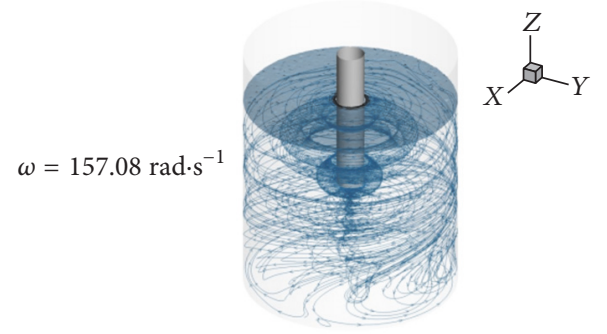

(m)

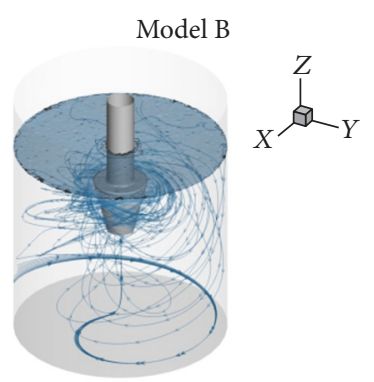

(b)

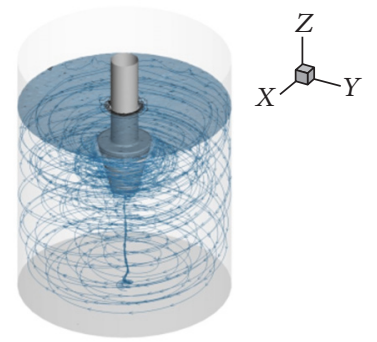

(e)

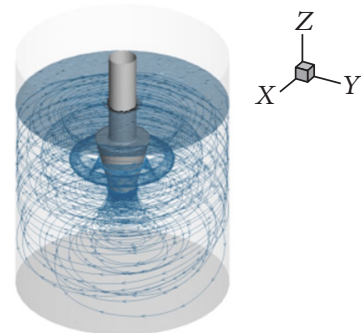

(h)

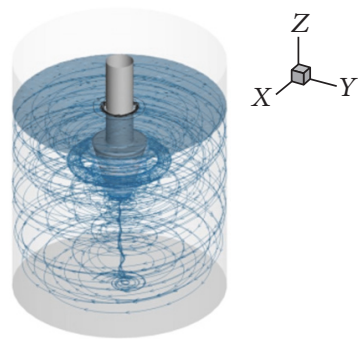

(k)

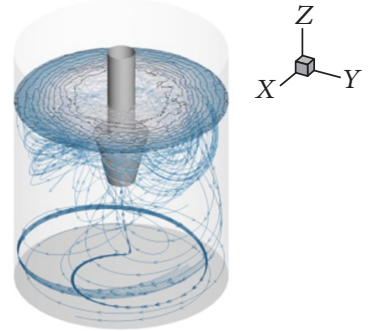

(n)

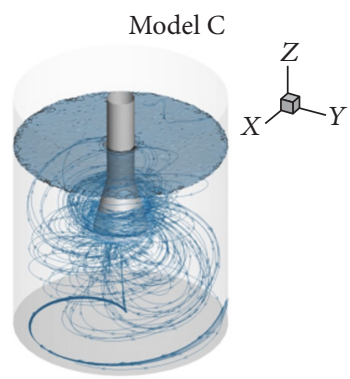

(c)

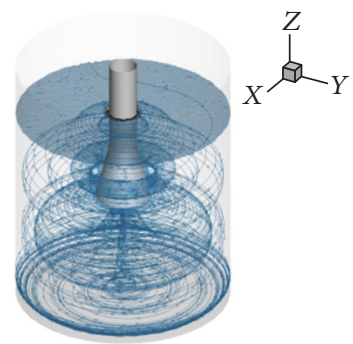

(f)

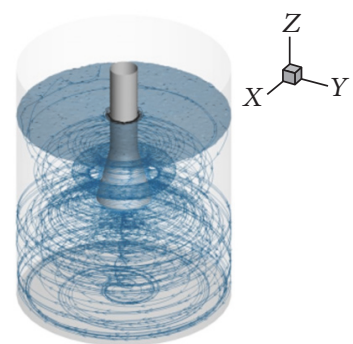

(i)

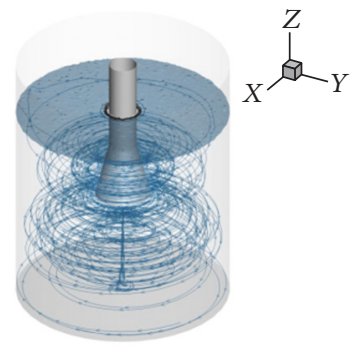

(l)

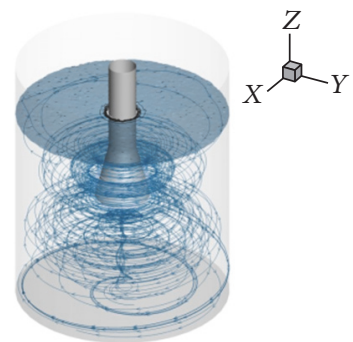

(o)

FIGURE 4: Velocity-blue-colored streamlines in three-dimensional numerical models at different rotation speeds. 
Method. The results were distributed in columns according to the model and in rows according to the rotation speed.

In model $\mathrm{A}$, it can be observed that circular structures are formed around the cylinder; they are divided into two as the speed of rotation increases. On the other hand, model $\mathrm{B}$ presents an irregular flow structure. As the rotation speed increases, the structure becomes symmetrical to the rotation axis of the cylinder. Model $\mathrm{C}$ presents larger structures than models $\mathrm{A}$ and $\mathrm{B}$, which ascend as the rotation speed increases.

At $\omega=20.94 \mathrm{rad} \cdot \mathrm{s}^{-1}$, model $\mathrm{C}$ has circular structures which are not symmetrical to the rotation axis of the cylinder though it is a more stable structure than those presented in model A and model B. The effect of the free surface is not considerable in the three cases, yet it begins to form a zone of low pressure around the cylinders. This effect causes the fact that, in the case of the models B and C, the structure presents nonsymmetric movements. Also, more streamlines around the tips are observed. In model $\mathrm{A}$, the streamlines are distributed along the cylinder, below the free surface. In models $\mathrm{B}$ and $\mathrm{C}$ it is possible to observe that there is a flow that passes near the base of the confined volume and ascends through the center of the system.

In model $\mathrm{A}$, at $\omega=62.83 \mathrm{rad} \cdot \mathrm{s}^{-1}$, there is a "coneshaped" flow that forms around the base of the cylinder and below the free surface. An area where the flow is fastened to the free surface can be noticed. It is also observed that there is an upstream flow below the cylinder and an irregular flow structure close to the base of the volume, which is not symmetrical.

In model A, a symmetrical flow pattern is observed; there is a concentration of streamlines in the inverted cone and a defined upward flow below the cylinder base. In model C, the flow is symmetrical around the rotation axis of the cylinder. However, the structures grow considerably. One structure is formed around the tip; a second structure is formed below the tip and occupies almost the whole diameter of the confined volume. There is also an upward flow in the center of the volume and below the base of the cylinder and a rotating flow in the same direction of the cylinder at the base of the volume.

The structures that are formed at this rotation speed are not mixed into each other. In the three cases, there is an upward flow below the cylinder. But still in model A, there are more streamlines, which indicates that there is a considerable flow that rotates in the same direction. In model $\mathrm{C}$, it is possible to observe at least one circular structure in the free surface, a second structure around the tip, a third structure around the toroid that forms outside the tip, a fourth circular structure below the base of the cylinder, and a fifth structure close to the lower wall.

In model $\mathrm{A}$, the cone-shaped structure observed at $\omega=$ $62.83 \mathrm{rad} \cdot \mathrm{s}^{-1}$ is completed and forms a spherical-shaped flow at a rotation speed of $\omega=94.25 \mathrm{rad} \cdot \mathrm{s}^{-1}$ and a coneshaped flow is defined below the base of the cylinder. The upward flow below the cylinder does not change the position considerably. Nevertheless, the flow has a swirl. The turbulent structures grow considerably and try to occupy the volume. There are a greater number of streamlines in the volume.

In model $\mathrm{B}$, the flow behavior maintains symmetry. A toroidal structure is formed and a conical structure also develops below the tip of the cylinder. The turbulent flow rises and is located around the cylinder, not below it.

For model C, the flow structures continue to grow below the cylinder. The toroidal structure is maintained around the cylinder. A conical shape flow around the cylinder is developed and promotes the deformation of the free surface. The flow below the base of the cylinder begins to rotate and move the rotation axis; this rotation moves the flow towards the lateral walls of the cylinder.

At $\omega=125.66 \mathrm{rad} \cdot \mathrm{s}^{-1}$, in model $\mathrm{A}$, the flow pattern is similar to the one for $\omega=94.25 \mathrm{rad} \cdot \mathrm{s}^{-1}$. Nonetheless, the cone-shaped flow below the cylinder is wider than the flow, as shown in Figure 4(g). The streamlines around the lateral walls spread along the volume and the spherical structure around the cylinder does not change considerably. At this rotation speed, the deformation of the free surface is greater.

For model B, the flow structure expanded along the volume. The upward flow is formed below the base of the cylinder and the toroidal structure around the tip is maintained as well.

At $\omega=125.66 \mathrm{rad} \cdot \mathrm{s}^{-1}$, the flow structure in model $\mathrm{C}$ is more symmetrical than the flow, as shown in Figure 4(i). The streamlines are not expanded to the volume and there is no greater interaction with the base of the volume. The toroidal structure around the tip is maintained. The upward flow no longer rotates in the same way as at previous rotation speeds.

In Figure $4(\mathrm{~m})$, the flow pattern for $\omega=157.08 \mathrm{rad} \cdot \mathrm{s}^{-1}$ is no longer symmetrical. Mainly, near the base of the volume, the swirl below the cylinder begins to rotate in the same direction as the solid. In addition, it deviates from the center of the volume. It is also observed that the streamlines around the cylinder promote a deformation of the free surface near the lateral walls of the cylinder. This phenomenon affects the stability of a quasi-stable flow behavior in the confined volume.

At this rotation speed, in Figure 4(n), the deformation of the free surface is considerable. The toroidal structure is also deformed considerably and a three-dimensional paraboloid flow is observed. The upward flow at the center of the volume changes the direction and continues to form a rotational flow in the base of the volume, but it is more intense than in previous speeds. Many of the interactions between the cylinder and the flow, also between the lateral walls and the flow, are considered higher. This phenomenon makes it difficult to separate the different interactions that could be presented and discussed due to the high rotation speed.

In Figure 4(o), the flow structure does not change considerably as the previous rotation speed, although it is possible to observe the rotation in the upward flow at the center of the volume, because the vortices are joining under the cylinder. The toroidal flow structure around the tip of the cylinder is not deformed.

\section{Conclusions}

In the present work, we studied the influence of three distinct types of cylindrical geometries of solids, which rotate at different speeds inside a cylindrical confined volume. Model 
A solid is a constant diameter cylinder, while in models B and $\mathrm{C}$ the tip sections were modified using the inverted and the right truncated cone geometries, respectively. These proposals were physically and numerically evaluated to understand the fluid-structure interaction at nonsteady condition.

In the physical experiments, the nonintrusive PIV technique was used to obtain the vector field in the central plane. This data was used to calculate the scalar kinetic energy in the plane. Afterwards, Line Integral Convolution was used to visualize the fluid motion in the plane. A time series was obtained after the calculation of the scalar kinetic energy, at every time, during the test.

In the studied system, a quasi-stable state, at nonsteady condition, requires that the working fluid maintains the same velocity magnitude around the volume. In this work, we represent the scalar kinetic energy data of the ideal flow condition with a uniform distribution plot at different constant rotation speed.

The kinetic energy data time series were presented in a normal probability plot to show the nonsteady nature of the fluid flow behavior in every rotation speed. Also, it allowed us to compare each of the models.

Numerical simulations allowed us to generate threedimensional streamlines of the turbulent flow in the confined volume. The experimental data obtained by PIV technique was qualitatively compared with the numerical results and was used as a validation technique. The numerical results support and complement the experimental data, showing the complex behavior of the hydrodynamics inside the confined volume.

Because of the numerous applications, model A solid geometry was considered as the reference model. It was found that models $\mathrm{A}$ and $\mathrm{C}$ generated a high turbulent flow inside the confined volume. Model B also presented a high turbulent flow. However, there is a quasi-symmetrical flow and stability at different rotation speeds that allow obtaining reproducibility in the flow pattern that could drive the velocity of different phenomena.

Experimental and numerical data on the rotating cylinder inside a cylindrical confined volume case are useful to characterize different operational conditions in numerous research and industry applications, for example, filtration systems, bioreactors, electrochemistry, and polymerization devices. The obtained results could be valid at different scales using a similar criterion.

\section{Conflicts of Interest}

The authors declare that they have no conflicts of interest.

\section{Acknowledgments}

Ignacio Carvajal-Mariscal, Cesar A. Real-Ramírez, Florencio Sánchez-Silva, and Jesús González-Trejo acknowledge the SNI for the distinction granted and the stipend received. Ignacio Carvajal-Mariscal acknowledge IPN and UAM for the sabbatical year. The physical experiments were developed in LABINTHAP SEPI-ESIME at the Instituto Politécnico
Nacional. The numerical simulations were developed in Laboratorio de Cómputo y Visualización Científica at Universidad Autónoma Metropolitana. This study was funded by Instituto Politécnico Nacional, Universidad Autónoma Metropolitana, and Consejo Nacional de Ciencia y Tecnología.

\section{References}

[1] A. Alexiadis, A. Cornell, and M. P. Dudukovic, "Comparison between CFD calculations of the flow in a rotating disk cell and the Cochran/Levich equations," Journal of Electroanalytical Chemistry, vol. 669, pp. 55-66, 2012.

[2] M. Carpinella, M. I. Velasco, E. V. Silletta, J. M. Ovejero, S. A. Dassie, and R. H. Acosta, "Determination of flow patterns in a rotating disk electrode configuration by MRI," Journal of Electroanalytical Chemistry, vol. 750, pp. 100-106, 2015.

[3] W. G. Cochran, "The flow due to a rotating disc," Mathematical Proceedings of the Cambridge Philosophical Society, vol. 30, no. 3, pp. 365-375, 1934.

[4] G. Eitelberg, "Weissenberg effect and its dependence upon the experimental geometry," Rheologica Acta, vol. 22, no. 2, pp. 131136, 1983.

[5] J. Gonzalez, C. Real, L. Hoyos, R. Miranda, and F. Cervantes, "Characterization of the hydrodynamics inside a practical cell with a rotating disk electrode," Journal of Electroanalytical Chemistry, vol. 651, no. 2, pp. 150-159, 2011.

[6] M. Prokop, R. Kodym, T. Bystron, M. Paidar, and K. Bouzek, "A rotating rod electrode disk as an alternative to the rotating disk electrode for medium-temperature electrolytes, Part I: The effect of the absence of cylindrical insulation," Electrochimica Acta, vol. 245, pp. 634-642, 2017.

[7] G. K. H. Wiberg and A. Zana, "Levich Analysis and the Apparent Potential Dependency of the Levich B Factor," Analytical Letters, vol. 49, no. 15, pp. 2397-2404, 2016.

[8] K. Blurton and A. Riddiford, "Shapes of practical rotating disc electrodes," Journal of Electroanalytical Chemistry (1959), vol.10, no. 5-6, pp. 457-464, 1965.

[9] P. Kiatkittikul, J. Yamaguchi, R. Taniki, K. Matsumoto, T. Nohira, and R. Hagiwara, "Influence of cationic structures on oxygen reduction reaction at Pt electrode in fluorohydrogenate ionic liquids," Journal of Power Sources, vol. 266, pp. 193-197, 2014.

[10] J. Dusting and S. Balabani, "Mixing in a Taylor-Couette reactor in the non-wavy flow regime," Chemical Engineering Science, vol. 64, no. 13, pp. 3103-3111, 2009.

[11] S. Dinarvand, "On explicit, purely analytic solutions of offcentered stagnation flow towards a rotating disc by means of HAM," Nonlinear Analysis: Real World Applications, vol. 11, no. 5, pp. 3389-3398, 2010.

[12] C. Y. Wang, "Off-centered stagnation flow towards a rotating disc," International Journal of Engineering Science, vol. 46, no. 4, pp. 391-396, 2008.

[13] Daichin and S. J. Lee, "Near-wake flow structure of elliptic cylinders close to a free surface: Effect of cylinder aspect ratio," Experiments in Fluids, vol. 36, no. 5, pp. 748-758, 2004.

[14] D. F. Kurtulus, F. Scarano, and L. David, "Unsteady aerodynamic forces estimation on a square cylinder by TR-PIV," Experiments in Fluids, vol. 42, no. 2, pp. 185-196, 2007. 
[15] N. A. Ozturk, A. Akkoca, and B. Sahin, "PIV measurements of flow past a confined cylinder," Experiments in Fluids, vol. 44, no. 6, pp. 1001-1014, 2008.

[16] L. Perret, "PIV investigation of the shear layer vortices in the near wake of a circular cylinder," Experiments in Fluids, vol. 47, no. 4-5, pp. 789-800, 2009.

[17] J. Vétel, A. Garon, and D. Pelletier, "Vortex identification methods based on temporal signal-processing of time-resolved PIV data," Experiments in Fluids, vol. 48, no. 3, pp. 441-459, 2010.

[18] A. Villegas and F. J. Diez, "Evaluation of unsteady pressure fields and forces in rotating airfoils from time-resolved PIV," Experiments in Fluids, vol. 55, no. 4, article no. 1697, 2014.

[19] W. Zhang, Daichin, and S. J. Lee, "PIV measurements of the near-wake behind a sinusoidal cylinder," Experiments in Fluids, vol. 38, no. 6, pp. 824-832, 2005.

[20] S. Jung, R. Kortlever, R. J. Jones et al., "Gastight Hydrodynamic Electrochemistry: Design for a Hermetically Sealed Rotating Disk Electrode Cell," Analytical Chemistry, vol. 89, no. 1, pp. 581585, 2017.

[21] I. Fluent, Fluent 6.3 Theory Guide, Ansys Inc, Canonsburg, Penn, USA, 2006.

[22] D. Dynamics, "DynamicStudio User's Guide," in Dynamic Studio. Dantec Dynamics, D. Dynamics, Ed., Dantec Dynamics, Skovlunde, Denmark, 2013, p. 660.

[23] K. S. Narendra and L. E. McBride, "Multiparameter SelfOptimizing Systems Using Correlation Techniques," IEEE Transactions on Automatic Control, vol. 9, no. 1, pp. 31-38, 1964.

[24] B. Cabral and L. C. Leedom, "Imaging vector fields using line integral convolution," in Proceedings of the 20th Annual Conference on Computer Graphics and Interactive Techniques (SIGGRAPH '93), pp. 263-270, August 1993.

[25] J. J. Filliben, "Probability plot correlation coefficient test for normality," Technometrics, vol. 17, no. 1, pp. 111-117, 1975. 


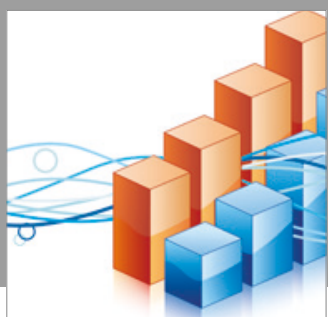

Advances in

Operations Research

vatersals

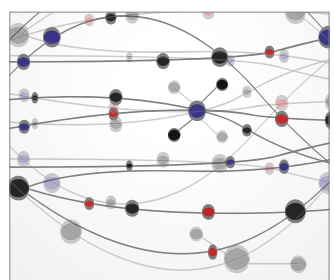

\section{The Scientific} World Journal
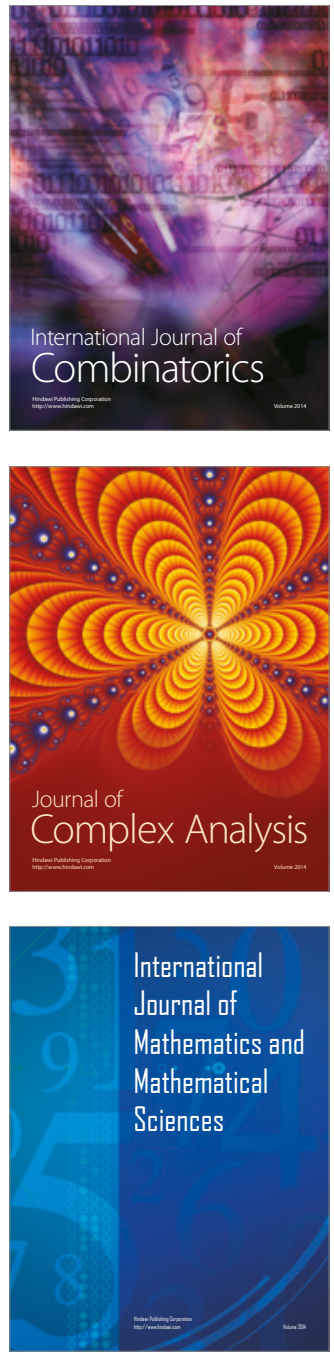
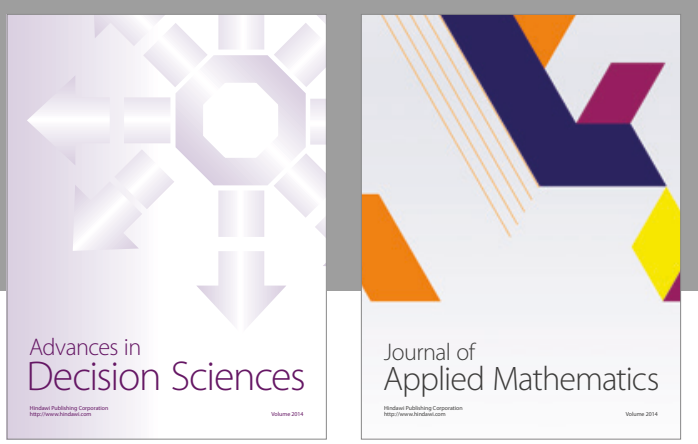

Algebra

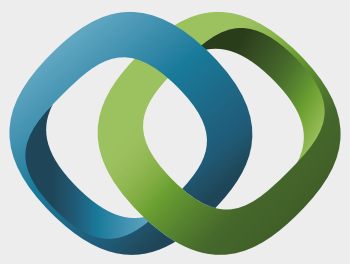

\section{Hindawi}

Submit your manuscripts at

https://www.hindawi.com
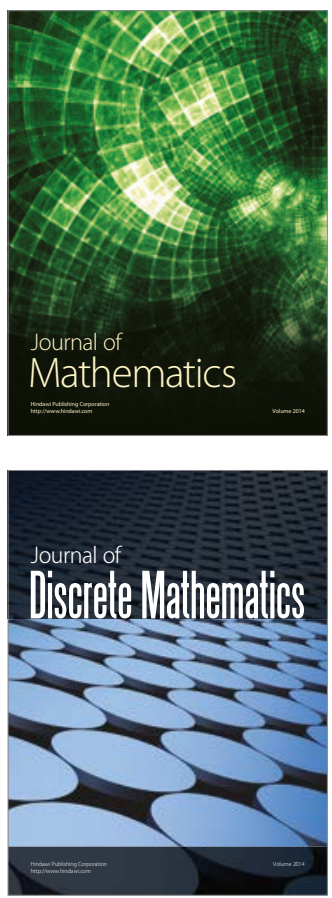

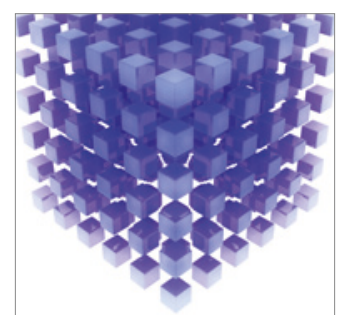

Mathematical Problems in Engineering
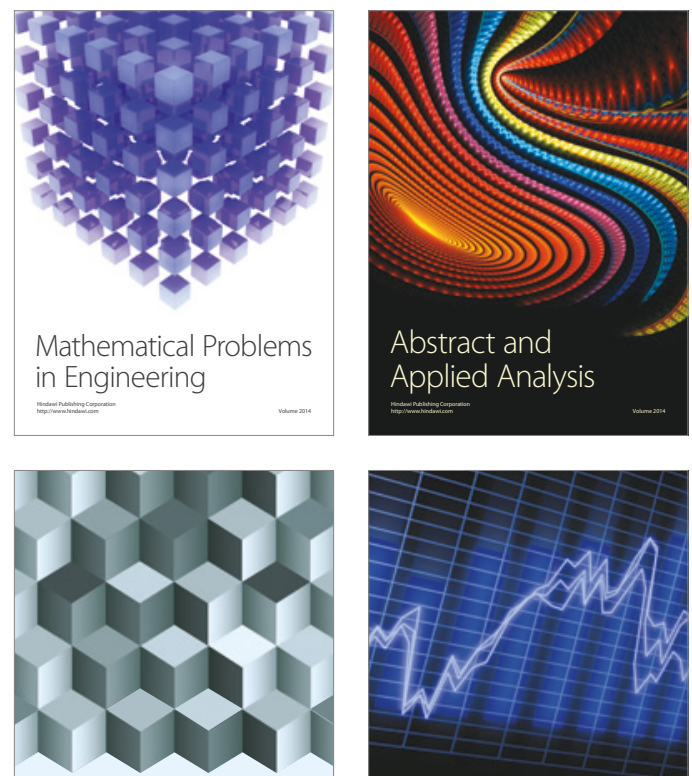

Journal of

Function Spaces

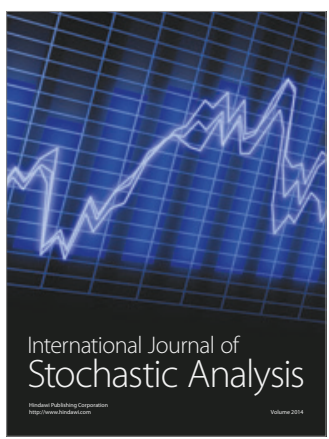

Probability and Statistics
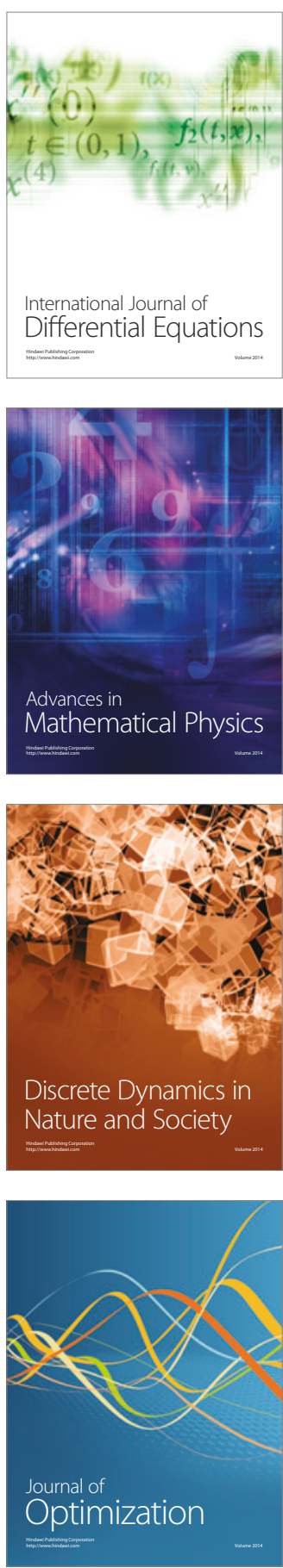